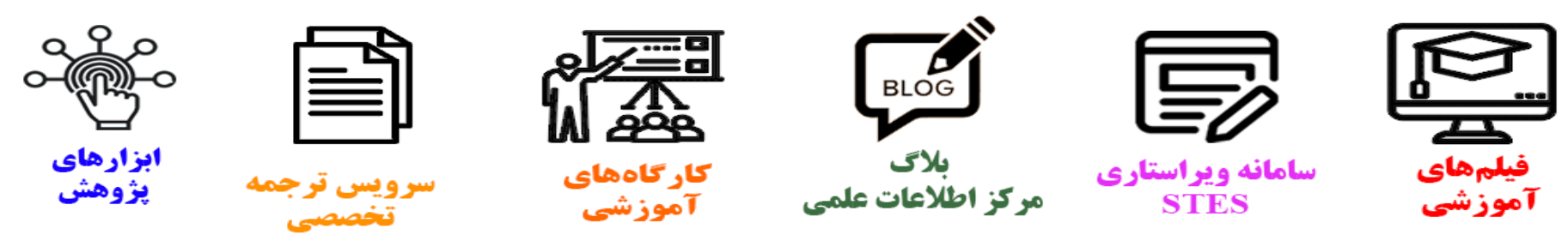

\title{
(c)
}

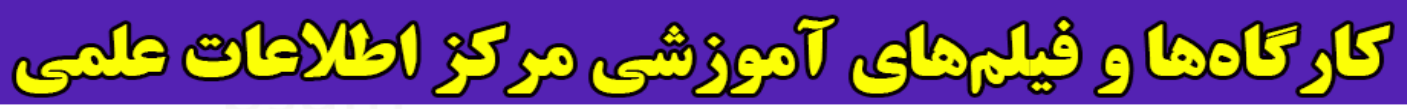
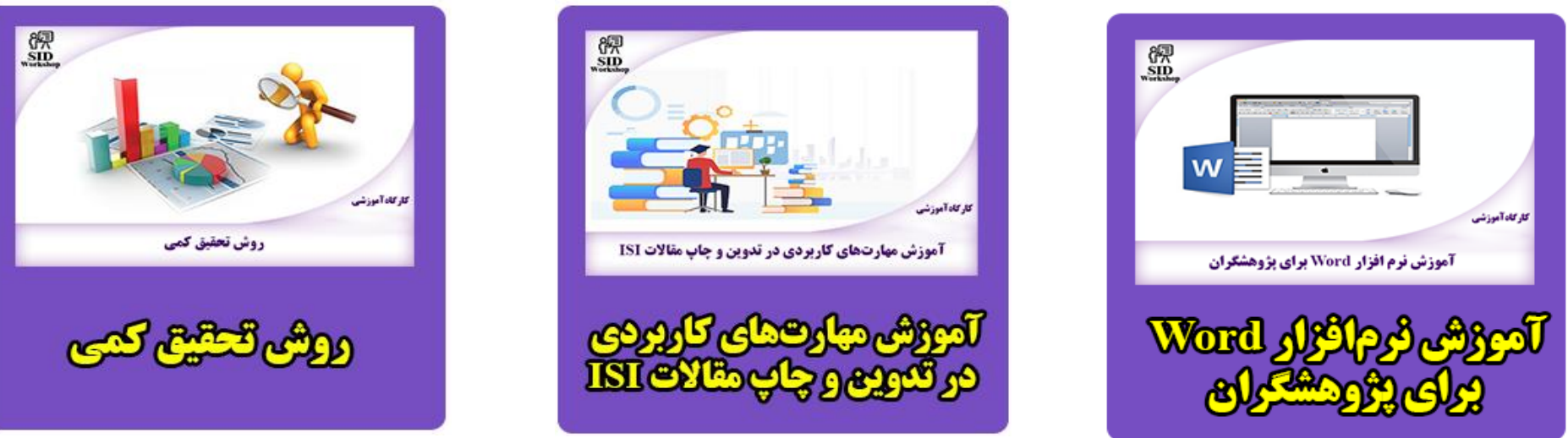


\title{
TECHNICAL NOTE
}

\section{PARETO OPTIMIZATION OF A TWO-DEGREE OF FREEDOM PASSIVE LINEAR SUSPENSION USING A NEW MULTI- OBJECTIVE GENETIC ALGORITHM}

\author{
A. Bagheri and M.J. Mahmoodabadi* \\ Department of Mechanical Engineering, University Guilan \\ P.O. Box 3756, Rasht, Iran \\ bagheri@guilan.ac.ir,mahmoodabadi@guilan.ac.ir \\ H. Rostami \\ Faculty of Mechanical Engineering, Islamic Azad University, Takestan Branch \\ Takestan, Iran. \\ hamidhr62@yahoo.com \\ Sh. Kheybari \\ Faculty of Industrial Engineering, Khatam University \\ Tehran, Iran. \\ shahram.kheibari@yahoo.com \\ *Corresponding Author
}

(Received: February 19, 2011 - Accepted in Revised Form: September 15, 2011)

doi: 10.5829/idosi.ije.2011.24.03a.08

\begin{abstract}
The primary function of the suspension system of a vehicle is to isolate the road excitations experienced by the tires and prevent them from being transmitted to the passengers. In this paper, we formulate an optimal vehicle suspension design problem with the quarter-car vehicle dynamic model. A new multi-objective genetic algorithm is used for Pareto optimization of a two-degree of freedom vehicle vibration model considering the two conflicting functions simultaneously. The important conflicting objective functions that have been considered in this work are, namely, sprung mass acceleration and relative displacement between sprung mass and tire. Comparison of the results with those reported in literature demonstrates the superiority of the presented method. It is shown that the results of 2-objective optimization provide more non-dominated choices for designers for optimal design of a product to select appropriate choices regarding the condition and purpose of using a vehicle.
\end{abstract}

Keywords Multi-objective Optimization, Genetic Algorithm, Pareto Design, Vehicle Vibration Model

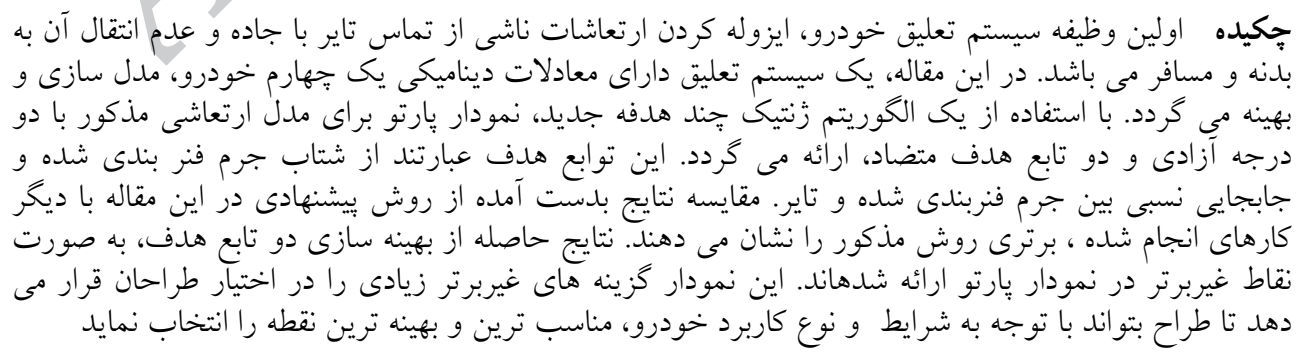

\section{INTRODUCTION}

Great amount of research activities during last decades has been directed to vibration control of machines run by engines. In particular, in automobiles, motions are influenced by the harmful 
effects of vibrations caused by engines and roads which have a pivotal role in driver's comfort. Griffin et al [1], Rakheja [2], and Barak [3] have shown that the interior vibration of a vehicle has a significant effect on comfort and road holding capability. To reduce this type of vibration, manufacturer's efforts have led to a suspension system installed between road excitation and vehicle body. Bouazara [4] studied the influence of suspension system parameters on the vibration of a model vehicle. In the same year, Hrovat [5] used a three-dimensional vibration model instead of the two-dimensional model to get more exact results. Crolla [6] applied a semi-active suspension model for improving the performance of vehicle. Bouazara and Richard [7] presented their vibration model in three-dimensional space demonstrating that this model has a good simulation of the vehicle behavior. Bouazara, et al [8] also studied three types of suspension systems (active, semi-active and passive) for an eight-degree of freedom vibration model. In that work, they combined all the performance criteria to form an objective function for a single-objective optimization process. For this purpose, they used weighting coefficients to adjust comfort and road holding capability criteria in the single-optimization design process. Gundogdu [9] presented an optimization of a four-degree of freedom quarter car seat and suspension system using genetic algorithms to determine a set of parameters to achieve the best performance of the driver's seat. The desired objective was proposed as the minimization of a multi-objective function formed by the combination of not only suspension displacement and tire deflection, but also the head acceleration and crest factor $(\mathrm{CF})$, which is not usually practiced by designers. Alkhatib, et al [10] applied genetic algorithm to the optimization problem of a linear one-degree of freedom (1-DOF) vibration isolator mount, and the method was extended to the optimization of a linear quarter car suspension model. The optimum solution was obtained numerically by utilizing GA and employing a cost function that sought minimizing absolute acceleration RMS (Root Mean Square) sensitivity to changes in RMS of relative displacement.

In fact, it is necessary for this type of the practical engineering applications to solve the optimization problems involving multiple design criteria. However, the design criteria of these practical engineering problems, which are called objective functions, may conflict with each other so that improving one of them will deteriorate the other. The inherent conflicting behavior of such objective functions lead to a set of optimal solutions named Pareto [11-14].

V. Pareto was the French-Italian economist who first developed the concept of multi-objective optimization in economics Pareto [15]. The concept of a Pareto front in the space of objective functions in multi-objective optimization problems (MOPs) stands for a set of solutions that are non-dominated to each other, but are superior to the rest of solutions in the search space. Evidently, changing the vector of design variables in such a Pareto optimal solutions consisting of these non-dominated solutions would not lead to the improvement of all objectives simultaneously. Consequently, such a change leads to a deterioration of at least one objective to an inferior one. Thus, each solution of the Pareto set includes at least one objective inferior to that of another solution in that Pareto set, although both are superior to others in the rest of the search space. The inherent parallelism in evolutionary algorithms makes them suitably eligible for solving MOPs.

The early use of the evolutionary search has been first reported in 1960s by Rosenberg [16]. Since then, there has been a growing interest in devising different evolutionary algorithms for MOPs. Amongst these methods, the vector-evaluated genetic algorithm (VEGA) [17], Fonseca and Fleming's genetic algorithm (FFGA) [13], strength Pareto evolutionary algorithm (SPEA) [18] and the Pareto-archived evolution strategy (PAES) [19] are the most important algorithms. A very good and comprehensive survey of these methods has been presented in [20,21]. Coello Coello has also presented an Internet-based collection of many papers as very good and easily accessible literature resources [22]. Basically, both NSGA and FFGA as Pareto-based approaches use the revolutionary nondominated sorting procedure originally proposed by Goldberg [23].

There are two important issues that have to be considered in such evolutionary multi-objective optimization methods: 1) driving the search towards the true Pareto optimal set, and 2) preventing premature convergence or maintaining the genetic diversity within the population [24]. The lack of 
elitism was also a motivation for modification of that algorithm to NSGA-II [25], in which a direct elitist mechanism, instead of a sharing mechanism, has been introduced to enhance the population diversity. This modified algorithm represents the state-of-the-art in evolutionary MOPs [26]. A comparison study between SPEA and other evolutionary algorithms on several problems and test functions showed that SPEA clearly outperforms the other multi-objective evolution algorithms [27]. Nariman-zadeh, et al applied a new multi-objective uniform-diversity genetic algorithm (MUGA) with a diversity preserving mechanism to multi-objective optimization of a five-degree of freedom vehicle vibration model [28].

In this paper, a new multi-objective uniformdiversity genetic algorithm with a diversity preserving mechanism called the $\varepsilon$-elimination algorithm is used for multi-objective optimization of a two-degree of freedom vehicle vibration model. The conflicting objective functions that have been considered for minimization are sprung mass acceleration $\left(\ddot{Z}_{2}\right)$, relative displacement between sprung mass and tire $\left(Z_{2}-Z_{1}\right)$. The design variables used in optimization of vibration are vehicle suspension damping coefficient $\left(\mathrm{C}_{2}\right)$ and vehicle suspension stiffness coefficient $\left(\mathrm{K}_{2}\right)$. Prominently, it is shown that a trade-off optimum design can be verified from those Pareto fronts obtained by multi-objective optimization process. Finally, the superiority of time domain vibration performance of such design point is shown in comparison with those given in the literature.

\section{PARETO MULTI-OBJECTIVE OPTIMIZATION}

Multi-objective optimization also called multicriteria optimization, or vector optimization has been defined as finding a vector of decision variables satisfying constraints to give acceptable values to all objective functions [25]. In general, it can be mathematically defined as:

Find the vector $X^{*}={ }^{\prime} x_{1}^{*}, x_{2}^{*}, \ldots, x_{n}^{*^{\prime} T}$ to optimize
$F(X)=\left\{f_{1}(X), f_{2}(X), \ldots, f_{k}(X)\right\}^{T} \quad$ subjected to $\mathrm{m}$ inequality constraints $g_{i}(X) \leq 0 \quad t=1,2, \ldots, m$ and $\mathrm{p} \quad$ equality constraints $h_{i}(X)=0 \quad j=1,2, \ldots, p$. Where $X^{*} \in R^{n}$ is the vector of decision or design variables, and $F(X) \in R^{n}$ is the vector of objective functions, each of which are to be minimized or maximized. However, without loss of generality, it is assumed that all objective functions are to be minimized. Such multi-objective minimization based on Pareto approach can be conducted using some definitions as:

Pareto Dominance: A vector $U=\left[u_{1}, u_{2}, \ldots, u_{n}\right]$, is dominant to vector $V=\left[v_{1}, v_{2}, \ldots, v_{n}\right]$ (denoted by $U \prec V$ ) if and only if

$\forall i \in\{1,2, \ldots, k\}, u_{i} \leq v_{i} \wedge \exists j \in\{1,2, \ldots, k\}: u_{i}<v_{j}(1)$

Pareto Optimality: A point $\mathrm{X}^{*} \in \Omega \quad(\Omega$ is a feasible region in $R^{n}$ ) is said to be Pareto optimal (minimal) if and only if there is not $\mathrm{X} \in \Omega$ which can dominate over $x^{*}$. Alternatively, it can be readily restated as:

$\forall \mathrm{X} \in \Omega, \mathrm{X} \neq \mathrm{X}^{*}, \exists \mathrm{i} \in\{1,2, . . \mathrm{k}\}: \mathrm{f}_{\mathrm{i}}\left(\mathrm{X}^{*}\right)<\mathrm{f}_{\mathrm{i}}(\mathrm{X})(2)$

Pareto Set: For a given multi-objective optimization problem (MOP), a Pareto set $\mathrm{P}^{*}$ is a set in the decision variable space consisting of all the Pareto optimal vectors:

$\mathrm{P}^{*}=\left\{\mathrm{X} \in \Omega \mid \gg \mathrm{X}^{\prime} \in \Omega: \mathrm{F}\left(\mathrm{X}^{\prime}\right)<\mathrm{F}(\mathrm{X})\right\}(3)$

Pareto Front: For a given MOP, the Pareto front $\mathrm{PT}^{*}$ is a set of vector of objective functions which are obtained using the vectors of decision variables in the Pareto set $\mathrm{P}^{*}$ that is:

$P T^{*}=\left\{F(X)=\left(f_{1}(X), f_{2}(X), \ldots, f_{k}(X)\right): X \in P^{*}\right\}(4)$

In other words, the Pareto front $\mathrm{PT}^{*}$ is a set of vectors of objective functions mapped from $\mathrm{P}^{*}$.

Evolutionary algorithms have been widely used for multi-objective optimization because of their natural properties suited for these types of problems. This is mostly because of their parallel or 
population-based search approach. Therefore, most of the difficulties and deficiencies within the classical methods in solving multi-objective optimization problems are eliminated. For example, there is no need for either several runs to find the Pareto front or quantification of the importance of each objective using numerical weights. In this way, the original non-dominated sorting procedure given by Goldberg [23] was the catalyst for several different versions of multi-objective optimization algorithms $[13,14]$. However, it is very important that the genetic diversity within the population be preserved sufficiently. This main issue in MOPs has been addressed by many related research works [24]. Consequently, the premature convergence of multi objective evolution algorithms is prevented and the solutions are directed and distributed along the true Pareto front if such genetic diversity is well provided. The Pareto-based approach of NSGA-II [25] has been used recently in a wide area of engineering multi-objective evolution algorithms because of its simple yet efficient non-dominance ranking procedure in yielding different levels of Pareto frontiers. However, the crowding approach is not efficient as a diversity preserving operator, particularly in problems with more than two objective functions [26].

\subsection{Multi-Objective Uniform-Diversity Genetic} Algorithm The multi-objective uniform-diversity genetic algorithm uses non-dominated sorting mechanism together with a $\varepsilon$-elimination diversity preserving algorithm to get Pareto optimal solutions of MOPs more precisely and uniformly.

2.2. The Non-Dominated Sorting Method The basic idea of sorting of the non-dominated solutions originally proposed by Goldberg [23], which has been used in different evolutionary multi-objective optimization algorithms, as in [25], has been adopted here. The algorithm simply compares each individual in the population with others to determine its non-dominancy. Once the first front has been found, all its non-dominated individuals are removed from the main population and the procedure is repeated for the subsequent fronts until the entire population is sorted and non-dominantly divided into different fronts.

A sorting procedure to constitute a front could be simply accomplished by comparing all the individuals of the population and including the nondominated individuals in the front.

It can be easily seen that the number of nondominated solutions in $\mathrm{P}^{*}$ grows until no further one is found. At this stage, all the non-dominated individuals so far found in $\mathrm{P}^{*}$ are removed from the main population and the whole procedure of finding another front may be accomplished again. This procedure is repeated until the whole population is divided into different ranked fronts. It should be noted that the first rank front of the final generation constitute the final Pareto optimal solution of the multi-objective optimization problem.

\subsection{The \&-Elimination Diversity Preserving} Approach In the $\varepsilon$-elimination diversity approach that is used to replace the crowding distance assignment approach in NSGA-II [25], all the clones and $\varepsilon$-similar indiyiduals are recognized and simply eliminated from the current population. Therefore, based on a value of as the elimination threshold, all the individuals in a front within this limit of a particular individual are eliminated. It should be noted that such $\varepsilon$-similarity must exist both in the space of objectives and in the space of the associated design variables. This will ensure that very different individuals in the space of design variables having $\varepsilon$-similarity in the space of objectives will not be eliminated from the population. The clones and $\varepsilon$-similar individuals are replaced from the population by the same number of new randomly generated individuals. Meanwhile, this will additionally help to explore the search space of the given MOP more effectively. It is clear that such replacement does not appear when a front rather than the entire population is truncated for $\varepsilon^{-}$ similar individual.

\section{MULTI-OBJECTIVE OPTIMIZATION OF VEHICLE VIBRATION MODEL}

A two-degree of freedom vehicle with passive suspension adopted from Ref. [4] is shown in Figure 1. This model is composed of one sprung mass joined to one unstrung mass (indicate tire and chassis). Moreover, the effect of degrees of freedom, linear motion (in vertical direction for sprung and unstrung masses), in terms of 
acceleration, velocity and movement, are considered in formulation of motion equations.

Parameters $\mathrm{M}_{1}, \mathrm{M}_{2}, \mathrm{~K}_{1}, \mathrm{~K}_{2}$, and $\mathrm{C}_{2}$ which denote the vehicle's fixed parameters are expressed as tire mass, sprung mass, tire stiffness coefficient, stiffness coefficient for vehicle suspension, and damping coefficient for vehicle suspension, respectively.

3.1. The Governing Dynamic Differential Equations of Motion The linear differential equations of motion with respect to the degrees of freedom are derived by the dynamic of this model and excited by a double bump as shown in Figure 2 .

Newton-Euler equations can be written as follows [4]:

$\mathrm{M}_{2} \ddot{\mathrm{Z}}_{2}=-\mathrm{K}_{2}\left(\mathrm{Z}_{2}-\mathrm{Z}_{1}\right)-\mathrm{C}_{2}\left(\dot{\mathrm{Z}}_{2}-\dot{\mathrm{Z}}_{1}\right)(5)$

$\mathrm{M}_{1} \ddot{\mathrm{Z}}_{1}=\mathrm{K}_{2}\left(\mathrm{Z}_{2}-\mathrm{Z}_{1}\right)+\mathrm{C}_{2}\left(\dot{\mathrm{Z}}_{2}-\dot{\mathrm{Z}}_{1}\right)-\mathrm{K}_{1}\left(\mathrm{Z}_{1}-\mathrm{Z}\right)(6)$

where $Z_{1}$ and $Z_{2}$ are vertical tire displacement and vertical displacement of the sprung mass, respectively. Further, $\dot{\mathrm{Z}}_{1}$ and $\dot{\mathrm{Z}}_{2}$ represent vertical tire velocity and vertical velocity of the sprung mass, respectively. $\ddot{Z}_{1}$ and $\ddot{Z}_{2}$ denote vertical tire acceleration and vertical acceleration of the sprung mass, respectively. Lastly, $\mathrm{Z}$ represents the excitation via road double bumps, as shown in Figure 2. The input values of fixed parameters are presented in Table 1 [4]. In this paper, $10000<\mathrm{K}_{2}<$ 16000 and $500<\mathrm{C}_{2}<2000$ are two design variables to be optimally found based on multiobjective optimization of two different objective functions, namely, sprung mass acceleration $\ddot{Z}_{2}$ and relative displacement between sprung mass and tire $\left(\mathrm{Z}_{2}-\mathrm{Z}_{1}\right)$.

\subsection{Two-Objective Optimization of Vehicle} Vibration Model In this section, the multiobjective uniform-diversity genetic algorithm presented in previous sections is used for multiobjective design of vehicle model shown in Figure 1. A population of 100 individuals with a crossover probability of 0.9 and mutation probability of 0.1 has been used in 1024 generations. It is clear from Figure 3 that obtaining a better value of one objective would normally cause a worse value of

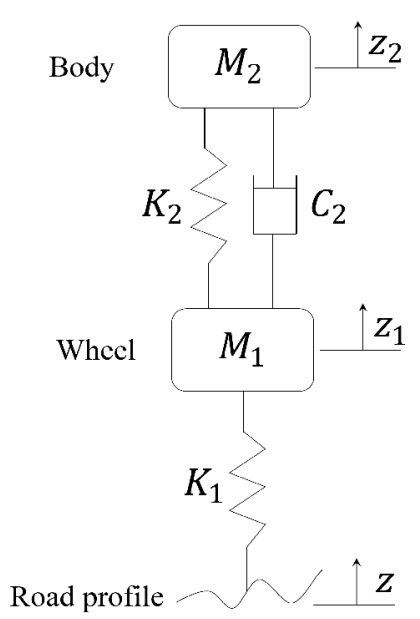

Figure 1. Vehicle vibration two-degree of freedom model with passive suspension adopted from Ref. [4].

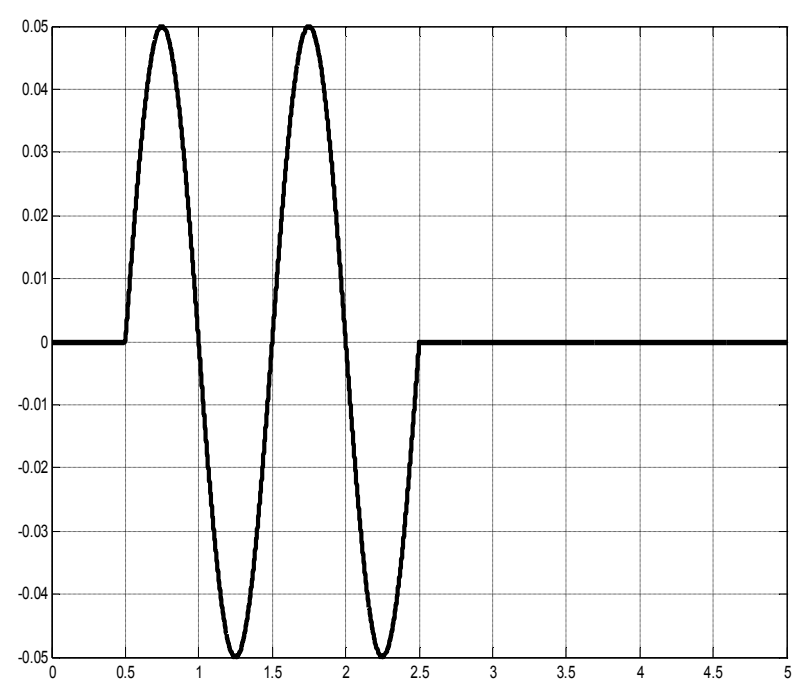

Figure 2. Double bumps excitation.

TABLE 1. The values of Fixed Parameters of the Model.

\begin{tabular}{cc}
\hline Parameters' Name & Parameters' Value \\
\hline $\mathrm{M}_{1}$ & $36 \mathrm{~kg}$ \\
$\mathrm{M}_{2}$ & $240 \mathrm{~kg}$ \\
$\mathrm{~K}_{1}$ & $160 \mathrm{kN} / \mathrm{m}$ \\
\hline
\end{tabular}


another objective. In other words, if a set of decision variables is chosen, the corresponding values of objectives will locate a point inferior to the corresponding Pareto front. Such inferior area in the space of the objective functions for Figure 3 is in fact top right sides.

Using NSGAII method and taking into consideration the two objective functions $\left(\mathrm{f}_{1}, \mathrm{f}_{2}\right)$, the result of multi-objective optimization named Pareto curve is shown in Figure 3. When compared to each other, it can be seen that all the points on Pareto curve are non-dominated.

Moving from point B with lowest relative displacement and highest chassis acceleration toward point A with lowest chassis acceleration and highest relative displacement on Pareto curve, objective function of chassis acceleration decreases, but that of relative acceleration will increase and vice versa.

As it is clear from the coordinate system of points in relation to objective function, in the face of selecting point $\mathrm{B}$ as optimal point and designing of suspension system using spring and damper coefficient according to point $\mathrm{B}$, we will have a vehicle with good handling. However, riding parameter will not be good, and when that vehicle passes over uneven places and obstacles on the road, high force and acceleration will be exerted to the driver and this is not desirable in production and use of that vehicle. The opposite is true for point $\mathrm{A}$.

Compared to points $\mathrm{A}$ and $\mathrm{B}$, the point $\mathrm{C}$ is positioned in better place. This could be a good position to select for designing the suspension system of vehicle, since there is no noticeable change from one objective function point of view, but the other objective function will become noticeably optimized. It worth to notice that each of the points in Pareto chart are related to $\mathrm{K}_{2}$ and $\mathrm{C}_{2}$ and obtained by exerting optimized parameters in equations performance. The system response will be obtained as time chart. Time performance curve for points $\mathrm{A}, \mathrm{B}$ and $\mathrm{C}$ as obtained from Pareto chart, and point D (as proposed in Ref. [4]) that stand for relative displacement, chassis acceleration, tire velocity, chassis velocity, tire displacement and chassis displacement, respectively, are shown in Figures (4-9).

Point D is taken from Ref. [4]. It is clear from Pareto chart in Figure 3 that point B is a better point from the handling point of view; A is good for

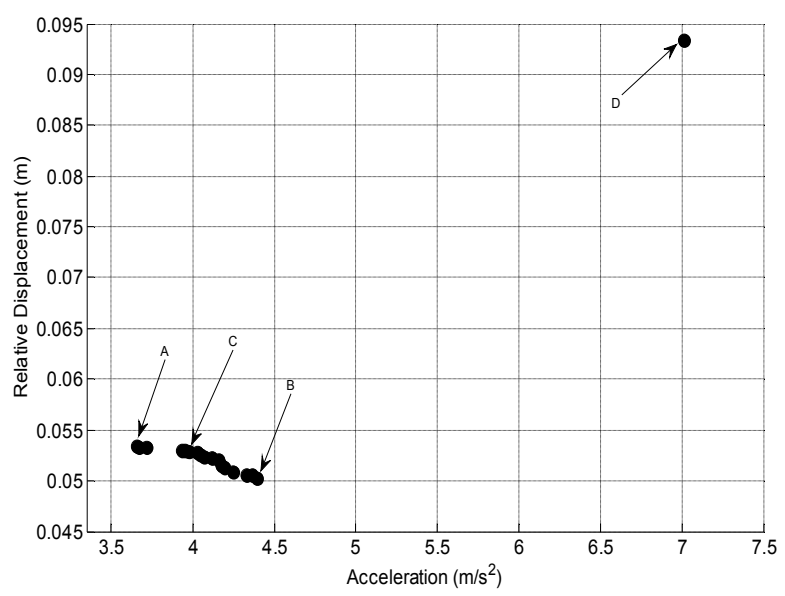

Figure 3. Pareto front.

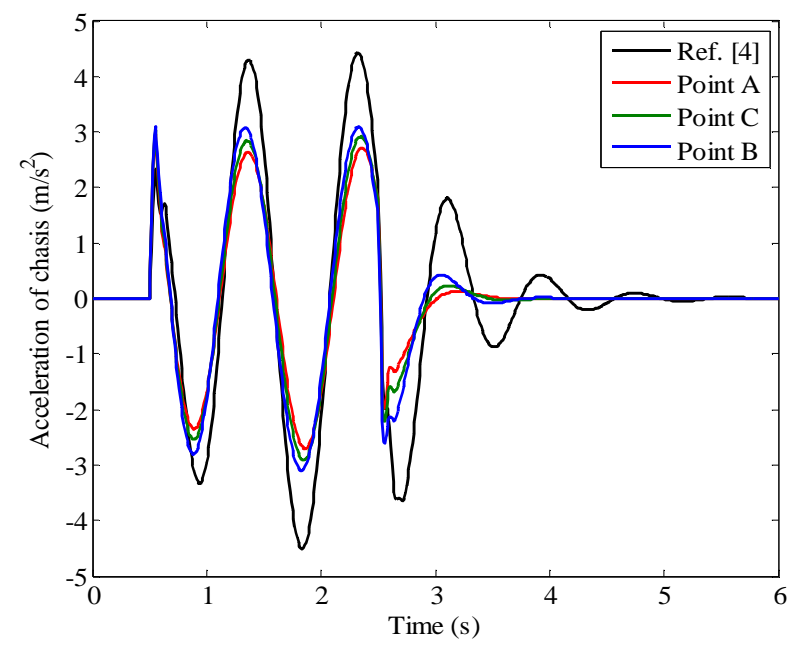

Figure 4. Chassis acceleration for optimum design points.

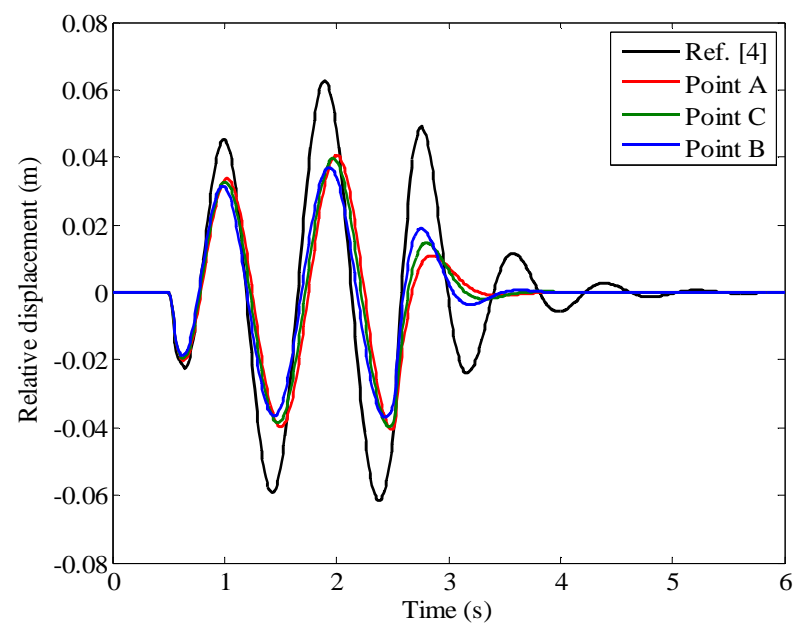

Figure 5. Relative displacement for optimum design points. 


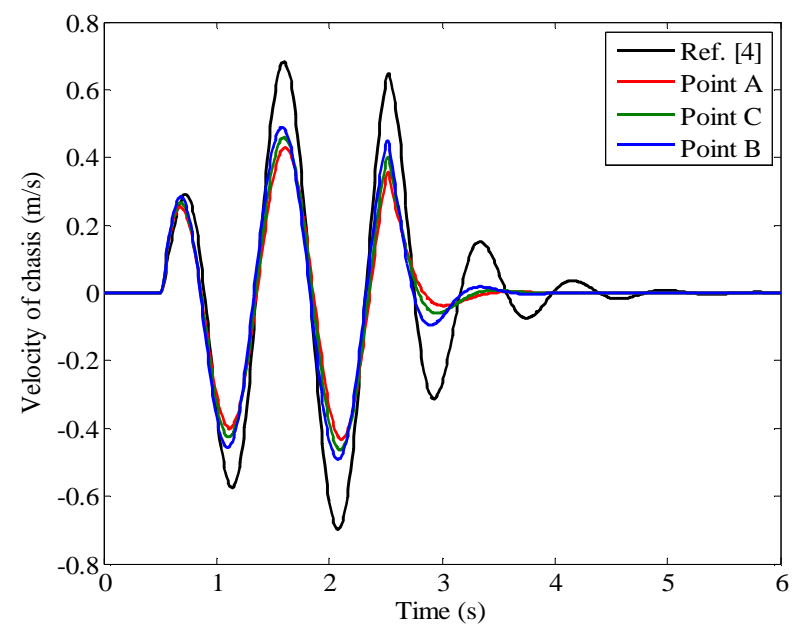

Figure 6. Chassis velocity for optimum design points.

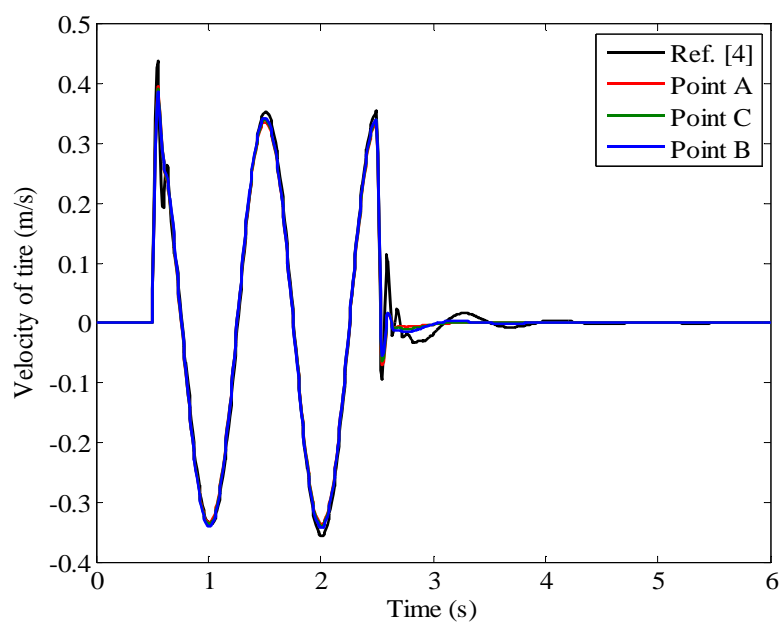

Figure 7. Tire velocity for optimum design points.

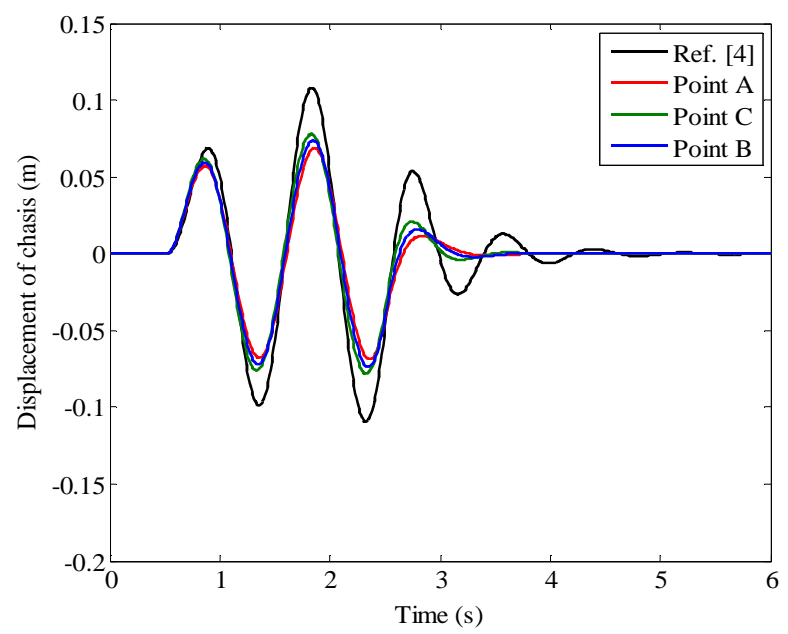

Figure 8. Chassis displacement for optimum design points. riding, but point $\mathrm{C}$ is the best point, and compared to point $\mathrm{D}$, it has better time performance from the riding and handling viewpoint. These figures and charts are clearly shown that all Pareto points have better time response regarding to one or both of the objective functions as compared to point $\mathrm{D}$.

Response of suspension system for each selected point from passing of the system over obstacle till the time of reaching its motionless position have been shown on the above mentioned charts. It is clear from the charts that suspension system with coefficients A, B and C obtained from Pareto chart has better time response and performance than point $\mathrm{D}$.

Corresponding values of points A, B, C, and D are shown in Table 2:

In Table 3 the maximums and minimums of objective functions are separately shown. It is obvious from Table 3 that relative displacement decreased from $93.85(\mathrm{~mm})$ in point $\mathrm{D}$ to 52.84 $(\mathrm{mm})$ in point $\mathrm{C}$ and chassis acceleration is decreased from $7.08654\left(\mathrm{~m} / \mathrm{s}^{2}\right)$ to $3.97995\left(\mathrm{~m} / \mathrm{s}^{2}\right)$.

\section{CONCLUSION}

Genetic algorithm optimization is a global optimization technique, searching for a design that minimizes an objective function subject to constraints. In this paper a multi-objective genetic algorithm with a recently developed diversity preserving mechanism called as NSGA-II, has been used to optimally design vehicle vibration model. The objective functions which conflict with each other were selected as sprung mass acceleration and relative displacement between sprung mass and tire. The multi-objective optimization of vehicle model led to the discovering of some important trade-offs among those objective functions. The superiority of the obtained optimum design points was shown in comparison with those reported in the literature. Such multi-objective optimization of vehicle model could unveil very important design tradeoffs between conflicting objective functions which would not have been found otherwise. Further, it has been shown that the results of two-objective optimization in terms of Pareto frontiers provide more choices for optimal design. These results are 


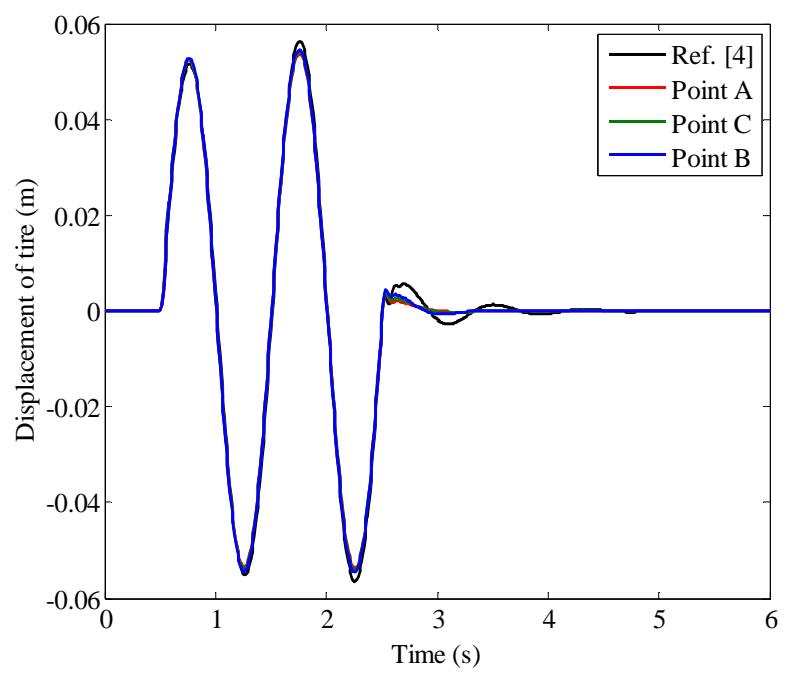

Figure 9. Tire displacement for optimum design points.

TABLE 2. Coefficients of Pareto Points.

\begin{tabular}{ccc}
\hline Point & $\mathrm{K}_{2}(\mathrm{~N} / \mathrm{m})$ & $\mathrm{C}_{2}(\mathrm{Ns} / \mathrm{m})$ \\
\hline A & 10000 & 1988.235 \\
B & 15529.41 & 2000 \\
C & 12305.88 & 2000 \\
D & 16000 & 1000 \\
\hline
\end{tabular}

TABLE 3. Parameters Related with Pareto Points.

\begin{tabular}{ccc}
\hline Point & $\begin{array}{c}\text { Relative } \\
\text { Displacement }(\mathrm{m})\end{array}$ & $\begin{array}{c}\text { Chassis } \\
\text { Acceleration }\left(\mathrm{m} / \mathrm{s}^{2}\right)\end{array}$ \\
\hline A & 0.05336 & 3.66035 \\
B & 0.05022 & 4.39457 \\
C & 0.05284 & 3.97995 \\
D & 0.09385 & 7.08654 \\
\hline
\end{tabular}

encouraging and suggest that GA can be easily used in other complex and realistic designs often encountered in the engineering.

\section{REFERENCES}

1. Griffin, M., Parsons, K. and Whitham, E., "Vibration and Comfort: Application of Experimental Results", Ergonomics, Vol. 25, (1982), 721-739.

2. Rakheja, S., "Computer-aided Dynamic Analysis and Optimal Design of Suspension Systems for Off-road Tractors", Ph.D. Thesis, Concordia University, Canada, (1985).

3. Barak, P., "Magic Numbers in Design of Suspensions for Passager Cars", Passager Car Meeting, Tennessee, (1991), 53-88.

4. Bouazara, M., "Etude Et Analyse De La Suspension Active Et Semi-Active Des Vehicules Routiers", Ph.D. Thesis, Universite Laval, Canada, (1997).

5. Hrovat, D., "Optimal Active Suspensions for 3D Vehicule Models", Proceedings of the American Control Conference, Arizona, U.S.A., Vol. 2, (1991), 1534-1541.

6. Crolla, D.A., "Semi-active Suspension Control for a Full Vehicule Model", SAE Technical Paper Series, (1992), 45-51.

7. Bouazara, M. and Richard, M. J., "An Optimal Design Method to Control the Vibrations of Suspensions for Passager Cars", International Mechanical Engineering Congress and Exposition, The Winter Annual Meeting of ASME, Atlanta, (1996), 61-68.

8. Bouazara, M. and Richard, M.J., "An Optimization Method Designed to Improve 3-D Vehicle Comfort and Road Holding Capability Through the Use of Active and Semi-active Suspensions", European Journal of Mechanics, Vol. 20, No. 3, (2001), 509-520.

9. Gundogd, D.E., "Optimal Seat and Suspension Design for a Quarter Car with Driver Model Using Genetic Algorithms", International Journal of Industrial Ergonomics, Vol. 37, No. 4, (2007), 327-332.

10. Alkhatib, R., Nakhaie Jazar, G. and Golnaraghi, M.F., "Optimal Design of Passive Linear Suspension using Genetic Algorithm", Journal of Sound and Vibration, Vol. 275, No. 3-5, (2004), 665-691.

11. Coello Coello, C. A. and Christiansen, A. D., "Multi Objective Optimization of Trusses Using Genetic Algorithms", Computers and Structures, Vol. 75, (2000), 647-660.

12. Coello Coello, C.A., Van Veldhuizen, D.A. and Lamont, G.B., "Evolutionary Algorithms for Solving MultiObjective Problems", New York, Kluwer Academic, U.S.A., (2002).

13. Fonseca, C.M. and Fleming, P.J., "Genetic Algorithms for Multi-Objective Optimization: Formulation, Discussion and Generalization", Proceedings of the Fifth International Conference on Genetic Algorithms, (1993), 416-423.

14. Srinivas, N. and Deb, K., "Multi-objective Optimization Using Non-Dominated Sorting in Genetic Algorithms", Evolutionary Computation, Vol. 2, No. 3, (1994), 221248.

15. Pareto, V., "In: Cours D'economic Politique", Rouge, Lausanne, Switzerland, (1896).

16. Rosenberg, R.S., "Simulation of Genetic Populations with Biochemical Properties", Ph.D. Thesis, University of Michigan, Ann Harbor, MI, U.S.A., (1967). 
17. Schaffer, J. D., "Multiple Objective Optimization with Vector Evaluated Genetic Algorithms", Proceedings of the First International Conference on Genetic Algorithms and Their Applications, Lawrence Erlbaum, London, U.K., (1985), 93-100.

18. Zitzler, E. and Thiele, L., "An Evolutionary Algorithm for Multi Objective Optimization: The Strength Pareto Approach", Technical Report 43, Computer engineering and communication network Lab, Swiss Federal Institute of Technology, Zurich, (1998).

19. Knowles, J. and Corne, D., "The Pareto Archive Devolution Strategy: a New Baseline Algorithm for Multi Objective Optimization", Proceedings of the Congress on Evolutionary Computation, IEEE Service Center, (1999), 98-105.

20. Coello Coello, C.A., "A Comprehensive Survey of Evolutionary Based Multi-objective Optimization Techniques", Knowledge and Information Systems, Vol. 1, No. 3, (1999), 269-308.

21. Khare, V., Yao, X. and Deb, K., "Performance Scaling of Multi-Objective Evolutionary Algorithms", Proceedings of Second International Conference on Evolutionary Multi-Criterion Optimization, Portugal, (2003), 376-390.

22. http://www.lania.mx/_ccoello/EMOO/.
23. Goldberg, D.E., "Genetic Algorithms in Search, Optimization, and Machine Learning", Addison-Wesley, Reading, MA, U.S.A., (1989).

24. Toffolo, A. and Benini, E., "Genetic Diversity as an Objective in Multi Objective Evolutionary Algorithms", Evolutionary Computation, Vol. 11, No. 2, (2003), 15167.

25. Deb, K., Agrawal, S., Pratap, A. and Meyarivan, T., “A Fast and Elitist Multi-Objective Genetic Algorithm: NSGA-II", IEEE Transactions on Evolutionary Computation, Vol. 6, No. 2, (2002), 182-197.

26. Coello Coello, C.A. and Becerra, R. L., "Evolutionary Multi Objective Optimization Using a Cultural Algorithm", Proceeding of the Intelligence Symposium, IEEE Service Center, Piscataway, (2003), 6-13.

27. Zitzler, E., Deb, K. and Thiele, L., "Comparison of Multi Objective Evolutionary Algorithms: Empirical Results", Evolutionary Computation, Vol. 8, No. 2, (2000), 173-175.

28. Nariman-Zadeh, N., Salehpour, M., Jamali, A. and Haghgoo, E., Pareto Optimization of a Five-Degree of Freedom Vehicle Vibration Model Using a MultiObjective Uniform-Diversity Genetic Algorithm (MUGA), Engineering Applications of Artificial Intelligence, Vol. 23, (2010), 543-551. 


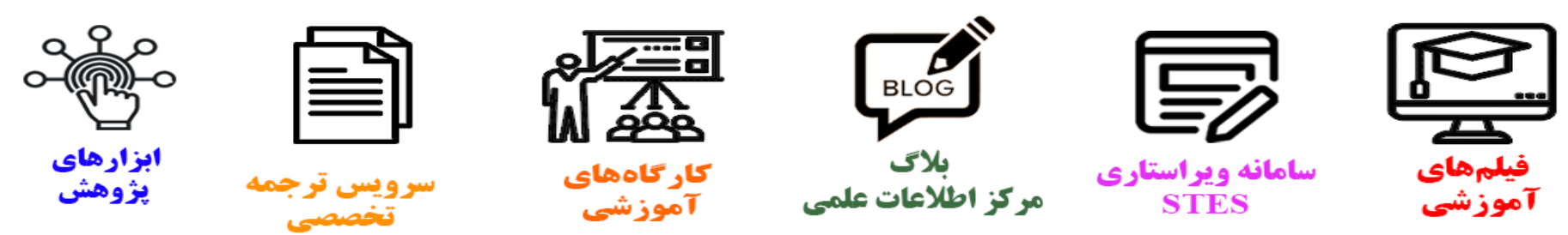

\section{(c)}

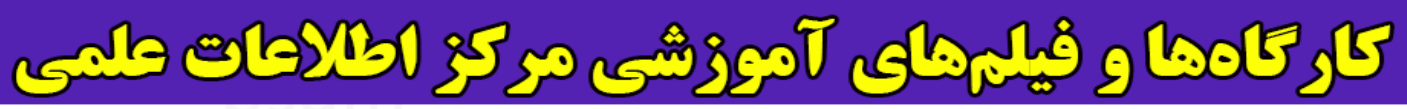
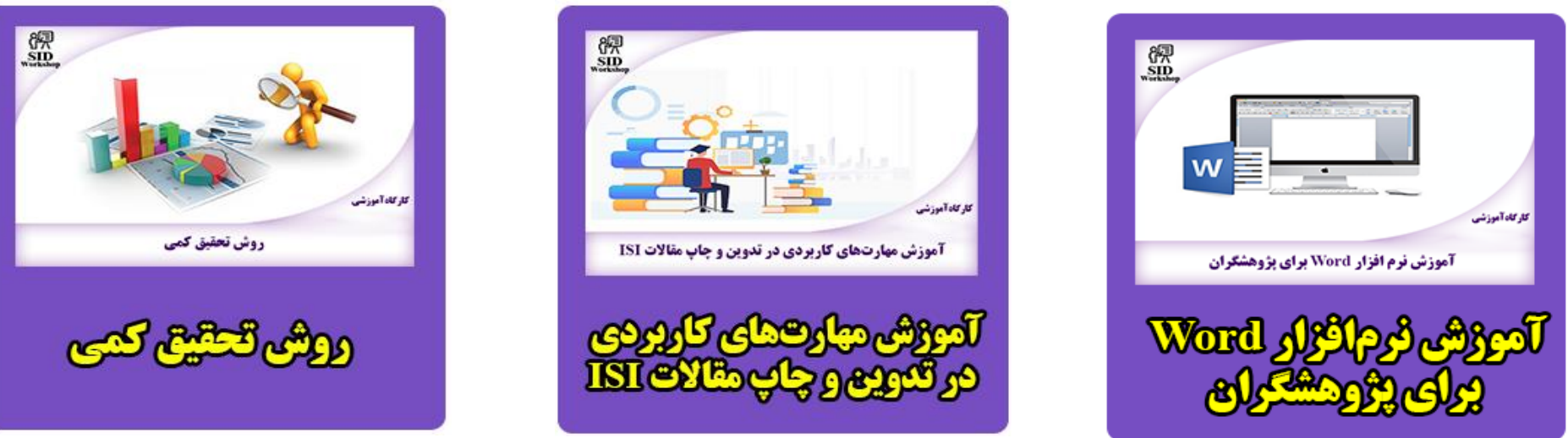